\title{
Motion-Based Games for Parkinson's Disease Patients
}

\author{
Oliver Assad, Robert Hermann, Damian Lilla, Björn Mellies, Ronald Meyer, \\ Liron Shevach, Sandra Siegel, Melanie Springer, Saranat Tiemkeo, Jens Voges, \\ Jan Wieferich, Marc Herrlich, Markus Krause, and Rainer Malaka
}

\author{
Digital Media, TZI, University of Bremen \\ sg10alletzi.de \\ http: //dm.tzi.de/sg10
}

\begin{abstract}
Games for rehabilitation are developing rapidly in recent years. It has been shown that utilization of therapy and gaming technology affects positively on the patients' physical and mental condition. However, to this day there are only few playable games for Parkinson's disease patients. This paper presents the development process of WuppDi! - a collection of five motion-based games for Parkinson's disease patients, aimed at supporting their exercises routines in various playful environments. We describe the game design challenges for Parkinson's disease patients and our solutions used in the games. Finally, we present the results of a conducted field test showing a very positive motivational effect among the majority of the patients but also highlighting remaining issues and technical difficulties, which can be beneficial for the future development in this field.
\end{abstract}

Keywords: serious games for health, parkinson's disease, motion-based game controls, physiotherapy.

\section{Introduction}

Parkinson's disease (PD) is a non-reversible, progressive neurodegenerative condition. The main physical symptoms of PD are slowness of movement, loss of ability of movement, rigidity of the arms, legs or trunk, resting tremor, and problems with balance and falling $[15,14]$. Besides the medication, physiotherapy plays the most important role in treating PD [6]. However, it is only effective when the exercises are done on an intensive daily basis [9, 17]. Daily training, supervised by a therapist, poses great economical problems for most patients $[5,12]$ and patients often get mentally bored and physically tired [20] thus losing their motivation. WuppDi!, our collection of motion-based games, addresses these problems by offering a low-cost game system to complement physiotherapy. Previous research has shown that games can be effective because exercising through games is perceived as play rather than therapy by the user [4]. Games offer the possibility of unlimited repetitions facilitated by a continuous feedback loop, immersion, and a state of winning [9]. While mainstream commercial training games and game devices like the Nintendo Wii could be useful for physical therapy purposes for PD patients, e.g., to help to decrease rigidity of patients with PD [10] or for lowering akinesia [9], improve coordination, spatial 
perception, reflexes, and balance [19], off-the-shelf games are not designed with the special requirements of PD patients in mind and thus are too difficult, especially for severe cases [9, 12, 17]. Furthermore, commercial games, e.g. for the Playstation EyeToy, usually include negative feedback when the performance of the player is not sufficient [4] and lack meaningful tasks in terms of therapy [8]. In contrast to these systems, WuppDi! is particularly designed for and with PD patients to aid them in exercising at home. Besides presenting our games, we also want to examine whether it is possible to let PD patients enjoy the exercises provided by our system.

\section{Related Work}

In the field of virtual systems for rehabilitation, the Interactive Rehabilitation EXercise (IREX) system is commonly referred to as the state of the art example [5, 12]. Unlike WuppDi!, this system requires an elaborate setup. In some cases, it also requires the users to wear gloves. Finally, due to its cost, the system is not applicable to home use. Based on studies of Kizony et al. the Playstation EyeToy, which shares a similar motion-based approach as WuppDi!, is able to induce the same level of felt presence as the more complex IREX system $[12,17]$.

A system similar to WuppDi! in terms of overall cost and input paradigms was developed by Burke et al. [5]. However, their system is designed to be used in the field of stroke rehabilitation. Their system and WuppDi! both rely on a webcam interface tracking colored markers. However, our system additionally offers games that can be played with free motion input where no colored markers are required.

A system in the area of PD therapy described recently by Yu et al. [20] makes use of a sophisticated motion capture system. While trying to catch falling objects, the player is shown an avatar mirroring his movements. Movements promoted by this system are of similar, repetitive nature as in our system. In contrast to WuppDi!, this system requires the user to wear motion tracking sensors placed on several positions on her body. To our knowledge there are only a few games specifically aimed at patients with PD sold commercially, none using full body motion input. One example produced in 2004 by Roche Pharma AG, called "Welt der Spiele", is a collection of mini-games using standard mouse and keyboard controls.

An early approach towards a taxonomy for games for rehabilitation considers features like application area, interaction technology, game interface, number of players, competitiveness, collaboration, genre, adaptability, progress monitoring, performance feedback and portability [18]. With regard to this taxonomy WuppDi! trains both cognitive and motor skills. The interaction with the interface is through motion tracking using 2D and 3D. Only one player is able to use the system at a time, but a turnbased competitive multiplayer mode is included. WuppDi! fits in the game genre "assorted". Currently, no dynamic adaptability is available but there are levels with various difficulties. Both visual and auditory feedback guides the player. However, there is no progress tracking. The game can be setup up at home as well as in clinics.

\footnotetext{
${ }^{1}$ http://www.slvideo.at/de/portfolio_interaktiv01.htm (accessed: April 18th, 2011)
} 


\section{WuppDi!}

\subsection{Development Method}

In order to develop games specifically tailored to PD patients, we followed a participatory design approach. We established a cooperation with the Bremen regional group of the "Deutsche Parkinson Vereinigung e.V." (German Parkinson Association), an association for self-help of PD patients, which provided us with contacts to patients and physiotherapists. With their help and by participating in physiotherapeutic sessions we gained further insights into patients' motion disabilities and training requirements. With respect to games and game technology, we started testing commercial off-the-shelf motion games (Wii Sports and Playstation Eye Toy Play) with patients to identify advantages and problems of game mechanics and input devices. The outcome was that participants in general enjoyed this new way of physical activity. However, these games turned out to be too demanding for this target group, especially in terms of handling the input device, visual overload and concerning speed and complexity of game play. The development of WuppDi! was initialized by creating throw-away prototypes for testing basic input mechanics. Afterwards, the final games were implemented in an iterative process over a period of 6 months, involving monthly field tests with small groups of PD patients for identifying problems and gaining feedback for constant enhancements. The final state was evaluated in a more extensive field study.

\subsection{Design Principles}

Through research and experiences during the development of the WuppDi! games the following principles were identified which should be taken into account when designing games for this target group.

Appropriateness of Movements - In order to design game mechanics that are beneficial for training, the movements encouraged by the game should be adapted to the abilities of the patients and ideally adopted from tailored gymnastic exercises. Especially the amplitude of movements is a crucial factor. Early prototypes showed that slow accurate movements of the arms are difficult and even induce or intensify tremors which cause frustration. Besides, PD patients tend to do very reduced movements and rather need to train continuous large amplitude movements ideally involving their whole body as also highlighted in the "Training BIG" approach [7]. The games should therefore foster wide fluent movements.

Positive Feedback - Visual and auditory feedback is an effective mechanism to support players' perception of their progress and the effect of their actions on game elements [5]. In the case of games for therapy and rehabilitation, especially positive feedback is of importance and should be emphasized [5, 8]. We experienced that patients felt frustrated when they could not achieve the goals relatively straight away. Some of them felt that the failure was a result of their own poor abilities and even

${ }^{2}$ http://www.parkinson-vereinigung.de 
excused for their performance. Therefore, it is important to reward players for their engagement, give them a feeling of success and encourage them to continue playing $[5,8]$ more so than in mainstream games. Besides encouraging sounds, nice visual effects, and rewarding feedback texts, we designed our games in a way that there is no failure in terms of loosing progress regardless of the player's performance.

Simplicity and Support - Many PD patients have, like elderly in general, little experience with computers and only few of them have played video games. Therefore, simplicity is an important principle to avoid excessive demand and frustration of players. Regarding gaming devices, our tests with the Wii revealed that patients are hardly able to coordinate moving a controller and pressing buttons at the same time. Even with no button input required, they seem confused by the presence of the buttons. We designed our input in a way that it only requires simple non-technical equipment or no equipment at all, respectively. For the game design it is important to avoid visual overload and respect the slower response times of the target group in terms of reaction and movement $[8,1]$. To help novel players overcome the barrier of first time use, it is beneficial to provide built-in instructions [1]. We created video tutorials for each mini-game, introducing story, goal, and controls of the game.

Adaptability - The cognitive abilities and motor skills of PD patients vary largely depending on their specific symptoms, the state of their disease, or even their daily condition. This poses challenges in designing a game play that is accomplishable but yet demanding and interesting enough, even for practiced players. Our collection of mini-games cover different degrees of complexity. While some games are rather simple and mainly focusing on movement, others require an advanced level of coordination or concentration. For alternating the difficulty within games, mechanisms from commercial games, such as levels, can be adopted [5]. Alternatively, approaches for dynamic adaptation of difficulty according to the player's performance and difficulty calibrations through physiotherapists have been proposed [2,5]. Each of our games features several levels or game modes of increasing difficulty which can be chosen by the player or an instructor.

Music and Rhythm - As described in the literature [13, 20, 16] and also observed in gymnastic sessions, music plays an important role in the training of PD patients for initializing and facilitating movements and overall enhancing the effectiveness of the therapy. Regarding these positive effects, music and rhythm should be considered important elements in game design. We suggest including background music with a clear beat that is aligned to the speed of game play. Beyond that, game mechanics can base upon rhythmic movements related to dancing. Here, the player scores higher when staying in the rhythm.

Appeal and Familiarity - One of the most challenging aspects in designing computer games for the elderly is to create appealing game scenarios. Understanding fields of interests, avoiding negative and violent contents can make the games more appealing for elderly to play [1]. Furthermore, Aison et al. [1] emphasize the importance of 
familiarity as the main motivation for game selection. Based on these elements, we chose fairytale as our main theme. Five well-known fairytales provide the background theme for each of our mini-games. The fairytale theme also offers the potential for cross-generational play of PD patients with their younger family members (e.g. grandchildren) which could increase their motivation to play.

\subsection{Implementation}

We aimed at a system that is deployable in physiotherapy groups or at homes of patients, i.e., that can be installed on prevalently existing hardware without the need of purchasing expensive or special equipment. Games are implemented using Microsoft XNA Game Studio 4.0 and can be run on any DirectX 9 compatible Windows PC. For the input, a webcam is required. While any webcam works in principle, we used the Sony PlayStation Eye camera due to its wide lens and good performance in low light conditions. For input processing, two alternative methods (with and without colored markers) were implemented using the OpenCV ${ }^{3}$ computer vision library Marker input is based on a simple color tracking approach that is able to distinguish yellow and red markers. The markers are graphically represented in the game, in the form of hands or tools, and can thus be used to interact with virtual game objects. As marker, we use self-made colored Styrofoam balls fixed on a wooden stick. Alternatively, colored gloves have been tested during development which turned out to be unpleasant for some of the patients. Due to a lack of fine motor skills they had problems putting the gloves on by themselves and had to be helped by the instructors. Marker-based games require the use of either one or two markers, one to hold in each hand. Markerless input is based on detecting movements of the player by computing the difference between subsequent frames of the webcam capture. Actions in the game are triggered if motion is detected within certain predefined areas. These are either invisible or displayed on screen, e.g. in the form of buttons or collectible items. In order to give the player spatial feedback, the webcam image or the binarized image difference is shown in the background during play.

\subsection{Games}

Following the design principles mentioned above, we created five mini-games that are depicted in figure 1. Each game is based on a well-known fairy tale. The games are accompanied by an interactive game menu (cf. figure 1, top left). The main game menu for selecting a mini-game contains six buttons arranged in an arch shape around a human silhouette. Seeing the webcam image in the background, the player can arrange herself to stand within the displayed outlines indicating the optimal position to reach all buttons. Using markerless input, buttons are triggered by reaching for them and waving the hand for three seconds. Once a game is chosen, a submenu displays a tutorial video and instructions. Subsequent menus, similarly structured as the main menu, let players select level, difficulty and (if applicable) number of players.

Cinderella is a markerless game that can be played by up to three players. The game encourages movements according to music rhythm. The player has to mirror the

\footnotetext{
${ }^{3}$ http://sourceforge.net/projects/opencvlibrary/
} 
movements of a prince avatar in the given rhythm and will thereby hit musical notes. Movements are based on gymnastic exercises and include: stretching both arms sideward and moving them up and down simultaneously, moving both arms diagonally up and down, and marching with swinging arms.

Ali-Baba is a single player memory game, played with two markers. The player has to get a secret treasure hidden behind a cave's door by memorizing and repeating motion sequences in the right order which are demonstrated by a thieve character. Stretching the arms towards buttons placed around the door triggers them and opens part of the door until the treasure is revealed. Town Musicians is a single player game, played with two markers representing a needle and a net, respectively. The player has to release captured town musicians' voices from flying bubbles by first freezing them with the net and afterwards bursting them with the needle. The game trains coordination between both arms and requires large continuous movements to reach bubbles across the screen.

Frog Prince is an explorative single player game based on markerless input in which the player has to steer a frog avatar through a pond, collect items and finally find a golden ball. The game requires wide circular arm movements, similar to breaststroke swimming. Synchronous movements of both arms make the frog swim forward; isolated single arm movements make it turn right or left.

Star Money is a single or multi player game that can be played with one or two markers. The game aims at training wide hand motion in a variety of ways depending on the selected game mode. In one, the player has to control a girl character by performing horizontal arm movements and collecting as many falling star coins as possible. In the other mode, she has to follow a star trail by performing fluent wide circular single arm movements in order to let stars fall from the sky. In multiplayer mode, both modes are combined, each played by one player.

\section{Field Study}

We conducted a field study to see how PD patients use the games in terms of game content, game play, and motion. The field study included a questionnaire, a reaction speed measurement tool, and an open interview part. Our field study was conducted in collaboration with local physiotherapy groups for PD patients with a total participant number of thirteen. Participants were between 54 and 86 years old with an average age of 70 and an average affection of PD of about 9 years. Both female (38\%) and male $(62 \%)$ participants participated. $70 \%$ of the participants reported to own a computer but none of them owned a webcam. $38 \%$ of all participants reported to play games that are included in operating systems, e.g., "Minesweeper" or "Solitaire". $15 \%$ had already played games on the Wii console. $46 \%$ of the participants had already been involved in the development process and were familiar with one of the previous versions of WuppDi! and familiar with the input paradigms. Eleven of 13 participants confirmed they do training at home where seven of them stated to do this on a daily basis. Only two of 13 participants negated to do any physical training at home. 

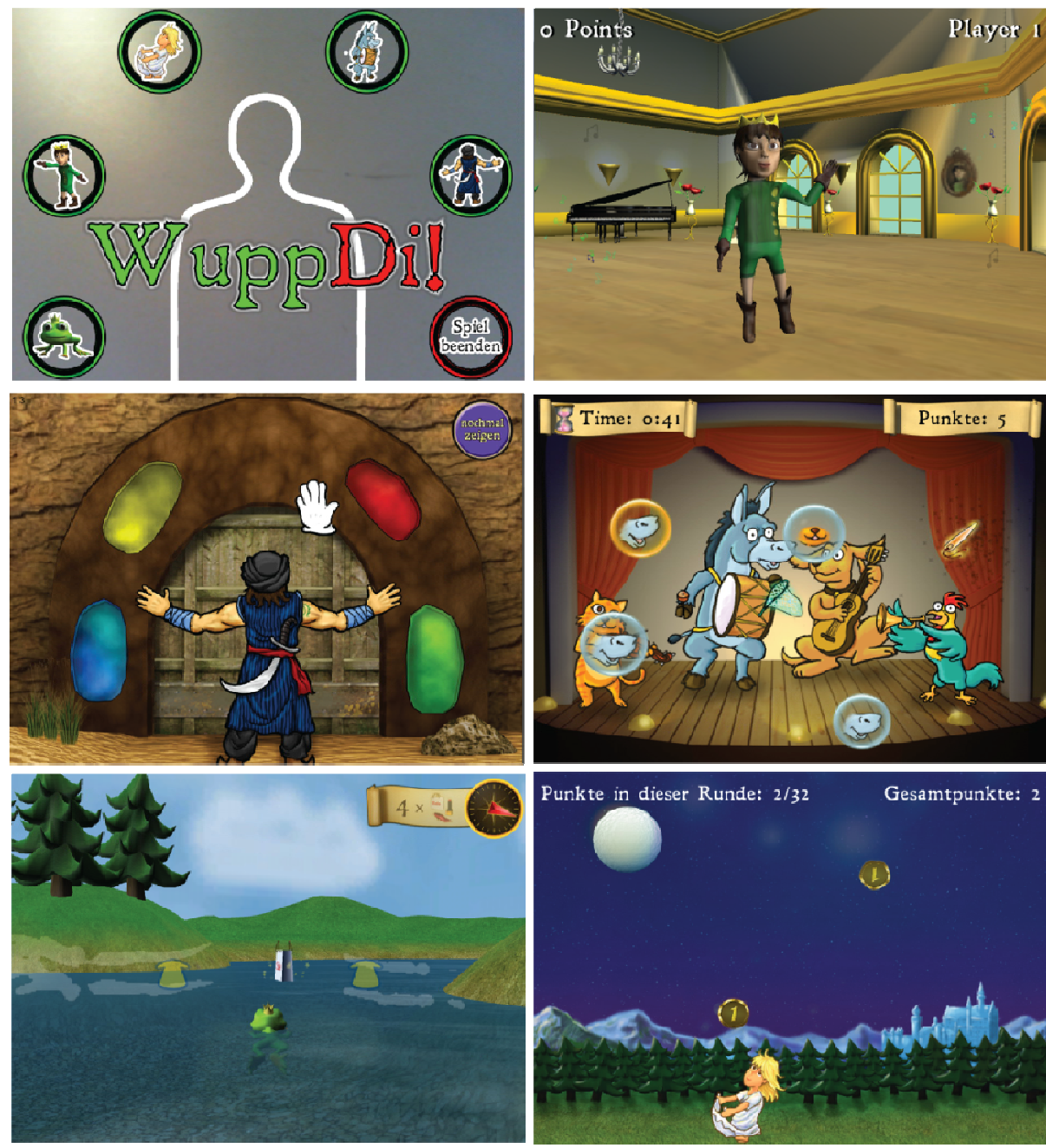

Fig. 1. Top left: Game Menu; Top right: Cinderella; Center left: Ali-Baba; Center right: Town Musicians; Bottom left: Frog Prince; Bottom right: Star Money

\subsection{Setup and Procedure}

We used a projector as a display device. Participants stood in front of the projection wall with enough space to perform the physical activities. We recorded the evaluation sessions on video from two different perspectives (front and back view of the participant). Each evaluation session began with the instructor reading a short introduction to the participant introducing the functionality of the reaction speed measurement tool (cf. figure 2). A short survey about demographical data followed and then the participants completed the reaction speed measuring tool. Each participant played two games covering both input methods used in WuppDi!. Each game was introduced by 
the instructor in terms of game play and game goal. Then the open interview was conducted. Afterwards, each participant left the evaluation room and completed the questionnaire part in private.

The participants were asked to complete a speed measuring test using our testing tool to assess their limits with regard to our chosen input method (cf. figure 2). The tool was designed to measure the reaction speed of a visual stimulus for activation of an in-game object. In the open interview directly after playing, participants were asked which game they liked and what specifically they had fun with. Then the participants were asked to fill out the questionnaire. With respect to the target group the overall number of questions we could ask in the questionnaire was severely limited. Therefore, we decided to aim for a broad feedback on game experience as well as usability considerations. Thus our questionnaire was composed of different parts of existing tools, sacrificing comparability to some extend for potential insights on a broader scale. The questionnaire was composed of parts of the Game Experience Questionnaire (GEQ) [11] and the System Usability Scale (SUS) [3]. We added some specific questions about physical motion, game theme, and aesthetics and asked the participants if they would play WuppDi! Together with their grandchildren. All questionnaire parts used a five point Likert scale. As the evaluation took place in a physiotherapist practical, experts on physical therapy were present during the evaluation sessions to monitor the participants while taking part in the sessions.

\subsection{Results and Discussion}

The results of the speed measurement tool showed that all participants performed equally and well in completing this task. The open interview showed the "Star Money" game was liked the most by the participants. Five of 13 participants mentioned that having success in the game was the main reason for having fun while playing. Three of 13 participants criticized they did not see a clear goal in the game. Overall our system scored 63 of 100 in the SUS part. The general outcomes of the categories "Positive Affect" and "Negative Affect" indicate that the majority of the participants had little or no negative affection (avg. 0.4 ; on a scale from $0=$ no affection to $4=$ big affection) while playing. Indeed, with the exception of one participant the results show an overall very positive affection (avg. 2.5; on a scale from $0=$ no affection to 4 = big affection). Video footage recorded during the evaluation suggests that this particular participant was in general very unconfident about game tasks and how to play the games. The GEQ average score was not significantly influenced by the score of this specific participant. The participants liked the game theme "fairy tales". Most of the participants considered to play WuppDi! together with their children or grandchildren. The aesthetics of the game were described as to be appealing. Positive influence on participants' motivation for exercising was also directly observed by attending physiotherapy staff. A participant was observed to do a stretching motion "even better and more dedicated" than in regular therapy to reach a specific goal in the "Star Money" game.

Game Menu - The navigation of the menu was observed to be intuitive as all participants understood how to navigate through it quickly and straightforward. However, usability problems could be observed in terms of the input paradigm as there were 
participants that accidentally executed menu items by tremor or unaware motion of their upper limbs.

Frog Prince - While the goal of "Frog Prince" was clear immediately, there were several usability and abstraction problems during game play. The participants had problems hitting the defined motion triggering areas in a synchronous way or keeping their whole body still to steer the frog left or right. The wider the participants were moving their arms the better did the input method work for the game. The unpredictable swim directions of the frog due to triggering motion areas unintentionally led to minor frustration experiences and detained the participants from completing the game goals in some cases. In contrast we had participants who understood the input method at once, hence reached all game goals and had fun playing the game.

Cinderella - The instruction to imitate a motion from a virtual game character was conversed also by people that never played a computer game before. Though, the exact imitation was observed to be difficult, especially in terms of timing to collect the items that appeared according to the rhythmical background music. Body motion of the participants was delayed frequently due to the observation process to imitate the motion of the game character which led to inferior performance in the game. Most participants could be observed to fulfill excessive upper limb stretching in order to collect game items that are in the upper screen area.

Star Money - The usage of markers on a wooden stick to control this game was easily adapted by most of the players. On the other hand side this caused some participants to do small and stiff arm motor activity. The auditory and visual feedback as well as the rhythmical music of the game was mentioned positively by almost all participants. In combination with the easy game mechanics and the easy to understand goal (collect the stars to gain points) most players considered "Star Money" as being the most fun game of WuppDi!.

Ali-Baba - The game goal of this game was unclear for most of the participants. The alternation of observing, memorizing and moving was considered as being difficult by most of them. The possibility to let the game character repeat the sequence of shown items was rarely used by the participants, therefore the activation button of this functionality was triggered unintentionally sometimes which led to confusion of the player. Confusion about the shown sequences and unintentional triggering of sequence repetitions were frustration factors for some participants.

Town Musicians - Observation of participants playing this game revealed a design issue: Marker symbols used in this game are not adequately salient as some of the participants were not able to recognize them instantaneously. Most of the participants could be observed to have a clear enhancement of ambition while exploring the game mechanics. Also the enhancement of reaction speed in terms of two hand coordination could be observed. Participants that did not show explorative behavior lost focus on the game goal which led to inferior performance of playing the game. 


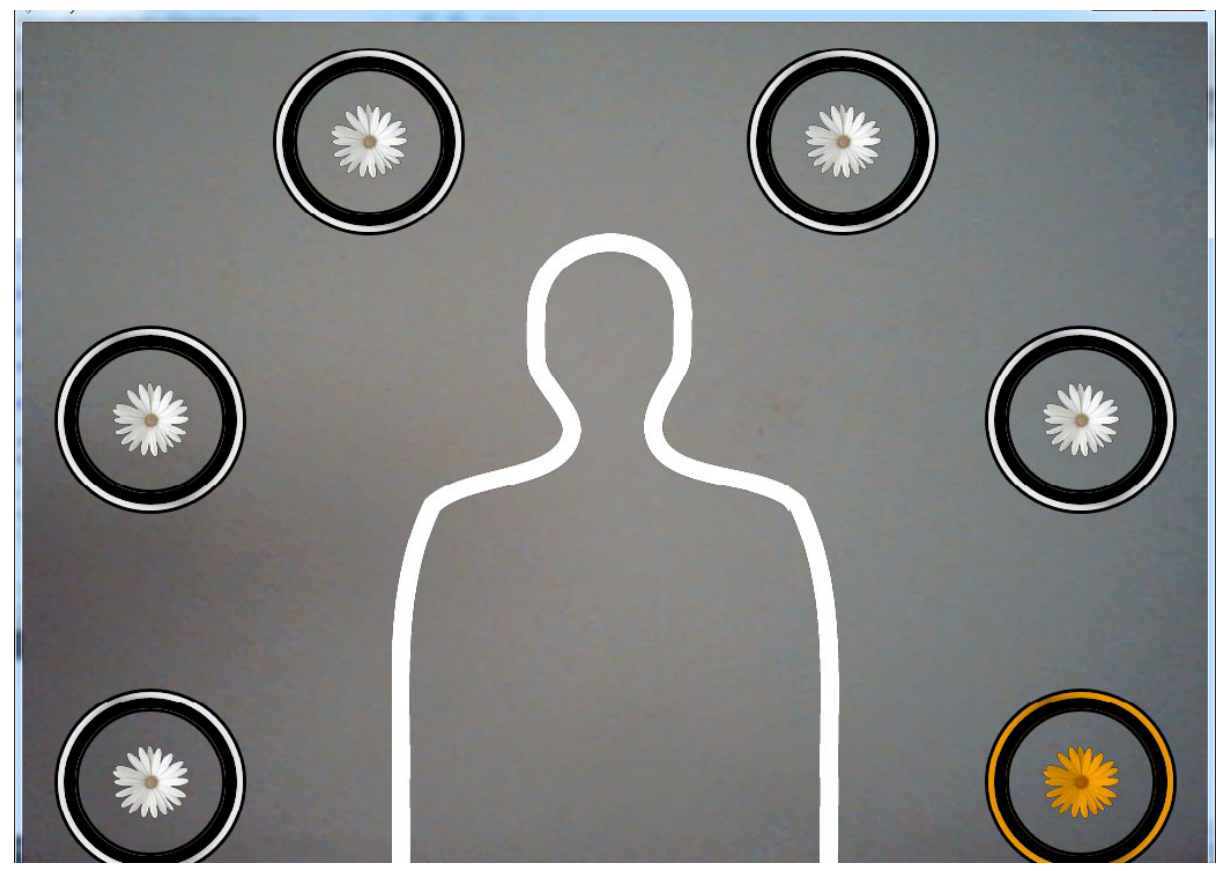

Fig. 2. Reaction time measurement tool

\section{Conclusion and Future Work}

The goal of our work was to examine how to develop a gaming system specifically for PD patients. For this, we used a participatory design approach and worked closely together with PD patients and physiotherapists. The intensive cooperation and the feedback of concerned persons proves the high interest of the target group in such a system and user tests during development as well as the final field study show that it is possible to let PD patients enjoy exercises in form of an Exergame. With WuppDi!, we developed a proof of concept games system consisting of five mini-games of different difficulty and focus. The overall usability of our system was rated positive according to the results of the SUS. Furthermore, results show that most of the patients enjoyed playing as indicated by the GEQ. We showed that it is possible to reach this level of enjoyment with the use of low cost technology like the Playstation Eye Camera.

The field study also confirms the design principles that were identified during the development process. Especially, the role of positive feedback and constant success for enjoyment were highlighted since participants liked those game most that they could all play successfully. Although following these principles throughout the development, the observations during the field study revealed that some of them are not sufficiently implemented. Here, we revealed a lot of potential for future work. In order to guarantee a satisfying game play for different patients, difficulty needs to be decreased in some of the games and a more dynamic method of adaption would be 
needed. Features like an initial calibration determining the maximum reach ability or a dynamic adaption of difficulty would also help to respect the player's physical abilities and help to achieve better results in therapy. Additionally more emphasis needs to be invested towards the fusion of game mechanics, music, and rhythm.

While initially aiming at a game system for home use, our results and observations show, that WuppDi! was not adequate for being used by single patients entirely on their own. On the one hand, still being in a prototype state, the system requires a setup that is rather complicated for the target group which is mostly not proficient in the use of computers. This minimally includes starting the software as well as attaching and positioning the webcam in an adequate way for playing. On the other hand, assistance of a healthy person is recommended for severe cases to assure balance and stability of the player. Rather, the system can be used in groups of patients supplementing gymnastics or physiotherapy. This also adds a positive social component to game play and allows for multiplayer playing.

Our field study is limited in expressiveness in terms of long term use and medical impact. In order to determine if the build in mechanics can help patients to reduce their physical symptoms, WuppDi! needs to be tested in a long term evaluation study. Additionally, doctors and physiotherapists need to use the tool and examine the outcomes in the terms of physical therapy usefulness. Lastly, adding the possibility to record a play session and sending the data to a therapist would greatly increase WuppDi!'s potential of being one day an engaging Serious Game for physiotherapy usable at home.

Acknowledgements. This work was supported by the Bremen regional group of the "Deutsche Parkinson Vereinigung e.V." and associated physiotherapists. We would like to thank them for their encouragement and constant feedback.

\section{References}

1. Aison, C., Davis, G., Milner, J., Targum, E.: Appeal and interest of video game use among the elderly. Harvard Graduate School of Education (2002)

2. Alankus, G., Lazar, A., May, M., Kelleher, C.: Towards customizable games for stroke rehabilitation. In: Proc. CHI 2010, Atlanta, USA, pp. 2113-2122 (2010)

3. Brooke, J.: SUS: a "quick and dirty" usability scale. In: Jordan, P.W., Thomas, B., Weerdmester, B.A., McClelland, I. (eds.) Usability Evaluation in Industry, pp. 189-194. Taylor \& Francis, London (1996)

4. Brooks, T., Petersson, E.: Play therapy utilizing the Sony EyeToy. In: The 8th Annual International Workshop on Presence, PRESENCE 2005, London, United Kingdom, pp. 303 $314(2005)$

5. Burke, J., McNeill, M., Charles, D., Morrow, P., Crosbie, J., McDonough, S.: Optimising engagement for stroke rehabilitation using serious games. The Visual Computer 25(2), 1085-1099 (2009)

6. Ebersbach, G.: BIG - spezifische Bewegungstherapie bei Parkinson

7. Farley, B., Koshland, G.: Training big to move faster: the application of the speedamplitude relation as a rehabilitation strategy for people with parkinson's disease. Experimental Brain Research 167(3), 462-467 (2005) 
8. Flores, E., Tobon, G., Cavallaro, E., Cavallaro, F.I., Perry, J.C., Keller, T.: Improving patient motivation in game development for motor deficit rehabilita-tion. In: Proc. ACE 2008, pp. 381-384. ACM, New York (2008)

9. Flynn, S., Palma, P., Bender, A.: Feasibility of using the Sony Playstation 2 gaming platform for an individual poststroke: a case report. Journal of Neurological Physical Therapy 31(4), 180-189 (2007)

10. Herz, N.B.: The Nintendo Wii and PD. PARKINSON Report 20(1), 7-8 (2009)

11. IJsselsteijn, W., de Kort, Y., Poels, K.: The Game Experience Questionnaire: Development of a self-report measure to assess the psychological impact of digi-tal games (2008) (manuscript in preparation)

12. Kizony, R., Weiss, P., Shahar, M., Rand, D.: Theragame - a home based vir-tual reality rehabilitation system. In: Proc. ICDVRAT 2006, pp. 209-214 (2006)

13. McIntosh, G.C., Brown, S.H., Rice, R.R., Thaut, M.H.: Rhythmic auditory-motor facilitation of gait patterns in patients with parkinson's disease. Journal of Neurology, Neurosurgery, and Psychiatry 62(1), 22-26 (1997)

14. National Collaborating Centre for Chronic Conditions. Parkinson's disease: na-tional clinical guideline for diagnosis and management in primary and secondary care. Royal College of Physicians, London (2006)

15. National Parkinson Foundation. What is Parkinson's disease (PD)? (2011), http://www.parkinson.org/Parkinson-s-Disease/

PD-101/What-is-Parkinson-s-disease (accessed April 11, 2011)

16. Pacchetti, C., Mancini, F., Aglieri, R., Fundar, C., Martignoni, E., Nappi, G.: Active music therapy in parkinson's disease: An integrative method for motor and emotional rehabilitation. Psychosomatic Medicine 62(3), 386-393 (2000)

17. Rand, D., Kizony, R., Weiss, P.L.: Virtual reality rehabilitation for all: Vivid GX versus Sony PlayStation II EyeToy. In: Proc. ICDVRAT 2004, pp. 87-94 (2004)

18. Rego, P., Moreira, P.M., Reis, L.P.: Serious games for rehabilitation: A survey and a classification towards a taxonomy. In: 5th Iberian Conference on Information Systems and Technologies (CISTI), pp. 1-6. IEEE, Los Alamitos (2010)

19. Wiemeyer, J.: Gesundheit auf dem Spiel? Serious Games in Prävention und Rehabilitation. Deutsche Zeitschrift für Sportmedizin 61(11), 252-257 (2010)

20. Yu, W., Vuong, C., Ingalls, T.: An interactive multimedia system for parkin-son's patient rehabilitation. In: MMSys 2010, Scottsdale, Arizona, USA (2010) 